\title{
Emergent parametric resonances and time-crystal phases in driven Bardeen-Cooper-Schrieffer systems
}

\author{
H. P. Ojeda Collado $\odot,{ }^{1,2,3,{ }^{*}}$ Gonzalo Usaj $\odot,{ }^{2,3}$ C. A. Balseiro, ${ }^{2,3}$ Damián H. Zanette $\odot,{ }^{2,4}$ and José Lorenzana $\odot^{1, \dagger}$ \\ ${ }^{1}$ ISC-CNR and Department of Physics, Sapienza University of Rome, Piazzale Aldo Moro 2, I-00185, Rome, Italy \\ ${ }^{2}$ Centro Atómico Bariloche and Instituto Balseiro, Comisión Nacional de Energía Atómica (CNEA)-Universidad Nacional de Cuyo \\ (UNCUYO), 8400 Bariloche, Argentina \\ ${ }^{3}$ Instituto de Nanociencia y Nanotecnología (INN), Consejo Nacional de Investigaciones Científicas y Técnicas (CONICET)-CNEA, \\ 8400 Bariloche, Argentina \\ ${ }^{4}$ Consejo Nacional de Investigaciones Científicas y Técnicas (CONICET), Argentina
}

(Received 28 July 2021; revised 15 October 2021; accepted 19 October 2021; published 10 November 2021)

\begin{abstract}
We study the out-of-equilibrium dynamics of a Bardeen-Cooper-Schrieffer condensate subject to a periodic drive. We demonstrate that the combined effect of drive and interactions results in emerging parametric resonances, analogous to a vertically driving pendulum. In particular, Arnold tongues appear when the driving frequency matches $2 \Delta_{0} / n$, with $n$ being a natural number and $\Delta_{0}$ being the equilibrium gap parameter. Inside the Arnold tongues we find a commensurate time-crystal condensate which retains the U(1) symmetry breaking of the parent superfluid/superconducting phase and shows an additional time-translational symmetry breaking. Outside these tongues, the synchronized collective Higgs mode found in quench protocols is stabilized without the need of a strong perturbation. Our results are directly relevant to cold-atom and condensed-matter systems and do not require very long energy relaxation times to be observed.
\end{abstract}

DOI: 10.1103/PhysRevResearch.3.L042023

Periodic driving of a many-body system allows the manipulation of the equilibrium phase diagram through phenomena such as dynamic localization [1-3] and the creation of new out-of-equilibrium states of matter such as time crystals [4-7] which exhibit time-translational symmetry breaking (TTSB). Related effects have been found in solids, often in connection with an enhanced stability of broken-symmetry phases [8-13]. These phenomena can be described using mathematical techniques developed by Floquet in the nineteenth century [14] and referred to as Floquet engineering [15].

An interesting Floquet engineering technique is to exploit parametric resonances and the associated parametric amplification allowing, for example, intrinsic losses to be overcome [16]. Quite generally, parametric resonances require a nonlinear medium whose intrinsic parameters can be modified periodically by a drive. This can be achieved in metamaterials as an array of Josephson junctions [17-19] or a structured superconducting waveguide [20]. Parametric resonances can also be generated in a single material such as, for example, a layered superconductor with intrinsic Josephson coupling between planes [21-23], a superconductor with surface allowed nonlinear coupling with the electromagnetic field [24], a charge-density wave [25] with nonlinear coupling between

\footnotetext{
*hector.pablo.ojedacollado@ roma1.infn.it

†jose.lorenzana@cnr.it

Published by the American Physical Society under the terms of the Creative Commons Attribution 4.0 International license. Further distribution of this work must maintain attribution to the author(s) and the published article's title, journal citation, and DOI.
}

amplitude and phase modes, or a semiconductor with nonlinear coupling between the electric field and phonons [26].

In this Letter we show that parametric resonances emerge naturally in any system whose dynamics is described by the Bardeen-Cooper-Schrieffer (BCS) Hamiltonian at times short with respect to the energy relaxation time (the so-called prethermal regime). Furthermore, we show that parametric resonances stabilize a discrete time-crystal phase [27-35] which retains the $\mathrm{U}(1)$ gauge symmetry breaking of the equilibrium BCS condensate.

We consider a weak-coupling fermionic condensate with $s$-wave pairing described by the BCS model and subject to a periodic drive that couples with the order parameter. The system is treated in the Anderson pseudospin formulation [36]. The Hamiltonian takes the form

$$
\hat{H}_{\mathrm{BCS}}=-2 \sum_{\boldsymbol{k}} \xi_{\boldsymbol{k}} \hat{S}_{\boldsymbol{k}}^{z}-\lambda(t) \sum_{\boldsymbol{k}, \boldsymbol{k}^{\prime}} \hat{S}_{\boldsymbol{k}}^{+} \hat{S}_{\boldsymbol{k}^{\prime}}^{-}
$$

Here, $\xi_{k}=\varepsilon_{k}-\mu$ measures the energy from the Fermi level $\mu$ and the pairing interaction is taken periodic in time with the driving strength $\alpha$ and the frequency $\omega_{d}$ as $\lambda(t)=\lambda_{0}[1+$ $\left.\alpha \sin \left(\omega_{d} t\right)\right]$. The modulation in the pairing interaction drives the system out of equilibrium. Possible realizations are discussed below and in Ref. [37]. The $\frac{1}{2}$-pseudospin operators are given by

$$
\begin{aligned}
& \hat{S}_{\boldsymbol{k}}^{x}=\frac{1}{2}\left(\hat{c}_{\boldsymbol{k} \uparrow}^{\dagger} \hat{c}_{-k \downarrow}^{\dagger}+\hat{c}_{-\boldsymbol{k} \downarrow} \hat{c}_{\boldsymbol{k} \uparrow}\right), \\
& \hat{S}_{\boldsymbol{k}}^{y}=\frac{1}{2 i}\left(\hat{c}_{\boldsymbol{k} \uparrow}^{\dagger} \hat{c}_{-\boldsymbol{k} \downarrow}^{\dagger}-\hat{c}_{-\boldsymbol{k} \downarrow} \hat{c}_{\boldsymbol{k} \uparrow}\right), \\
& \hat{S}_{\boldsymbol{k}}^{z}=\frac{1}{2}\left(1-\hat{c}_{\boldsymbol{k} \uparrow}^{\dagger} \hat{c}_{\boldsymbol{k} \uparrow}-\hat{c}_{-\boldsymbol{k} \downarrow}^{\dagger} \hat{c}_{-\boldsymbol{k} \downarrow}\right),
\end{aligned}
$$


and $\hat{c}_{\boldsymbol{k} \sigma}^{\dagger}\left(\hat{c}_{\boldsymbol{k} \sigma}\right)$ is the usual creation (annihilation) operator for fermions with momentum $\boldsymbol{k}$ and spin $\sigma$. The operator $\hat{S}_{\boldsymbol{k}}^{ \pm} \equiv \hat{S}_{\boldsymbol{k}}^{x} \pm i \hat{S}_{\boldsymbol{k}}^{y}$ creates or annihilates a Cooper pair $(\boldsymbol{k},-\boldsymbol{k})$. Due to the all-to-all interaction in Eq. (1), a mean-field treatment becomes exact in the thermodynamic limit and the dynamics can be obtained by solving for each pseudospin in a self-consistent field. The BCS mean-field Hamiltonian can be written as $\hat{H}_{\mathrm{MF}}=-\sum_{\boldsymbol{k}} \hat{\boldsymbol{S}}_{\boldsymbol{k}} \cdot \boldsymbol{b}_{\boldsymbol{k}}$, where $\boldsymbol{b}_{\boldsymbol{k}}(t)=$ $\left(2 \Delta(t), 0,2 \xi_{k}\right)$ represents an effective magnetic field vector for the $\frac{1}{2}$-pseudospin operator $\hat{S}_{k}=\left(\hat{S}_{k}^{x}, \hat{S}_{k}^{y}, \hat{S}_{k}^{z}\right)$. Here, without loss of generality, we have assumed a real equilibrium BCS order parameter $\left(\Delta_{0}\right)$, a condition that remains valid over time due to electron-hole symmetry. The instantaneous BCS order parameter is given by

$$
\Delta(t)=\lambda(t) \sum_{k} S_{k}^{x},
$$

where symbols without a hat denote the expectation value of operators in the time-dependent BCS state.

At equilibrium, in the absence of periodic perturbations, the $\frac{1}{2}$-pseudospins align in the direction of their local fields $\boldsymbol{b}_{\boldsymbol{k}}^{0}=\left(2 \Delta_{0}, 0,2 \xi_{\boldsymbol{k}}\right)$ in order to minimize the system's energy. This is used as an initial condition and once the pairing interaction is modulated, the pseudospins evolve in time obeying the equation of motion $\frac{d \boldsymbol{S}_{k}}{d t}=-\boldsymbol{b}_{\boldsymbol{k}}(t) \times \boldsymbol{S}_{\boldsymbol{k}}(\hbar \equiv 1)$. In contrast with Refs. [22,37,38], we focus on subgap frequencies $\left(\omega_{d} \leqslant\right.$ $\left.2 \Delta_{0}\right)$ and weak driving amplitude $(\alpha<0.2)$. We consider $N=10^{4}$ pseudospins equally spaced in energy $\xi_{\mathbf{k}}$, within an energy range of $W=40 \Delta_{0}$ around $\mu$ [39].

To characterize the dynamical phase transitions (DPT), we use $\bar{\Delta}$ as the dynamical order parameter, defined as the average of the order parameter $\Delta(t)$ over a large time window in the stationary regime. Figure 1(a) shows a map of $\bar{\Delta}$ as a function of the driving strength $\alpha$ and the driving frequency $\omega_{d}$. There are two distinct main regions in the phase diagram, one in which $\bar{\Delta} \approx 0$ (green area), and another one in which the temporal average is close to the initial equilibrium value $\Delta_{0}$ (orange area). We see resonant behavior (i.e., the average order parameter close to zero), each time the drive frequency matches $\omega_{d}=2 \Delta_{0} / n$, with $n$ being a natural number, within a region that becomes larger as the driving strength $\alpha$ grows, forming so-called Arnold tongues. Since these features emerge by varying an internal parameter of the system, they may be called parametric resonances. Even more substantially, the phase diagram is remarkably similar to that in Fig. 1(c) corresponding to the archetypal model of a parametric oscillator, namely, a vertically forced pendulum with a pump frequency, $\omega_{p}$, as shown schematically in Fig. 1(b). This analogy requires the following: (i) identification of the natural frequency as $\omega_{0}=2 \Delta_{0}$, (ii) identification of the pump frequency of the pendulum as $\omega_{p}=2 \omega_{d}$, (iii) inclusion of a small damping constant $\eta$ in the pendulum [39], and (iv) identification of the regions with large deviation from equilibrium in the pendulum (resonances) with the regions of zero average gap. Thus, the usual parametric resonances at $\omega_{p}=2 \omega_{0} / n$ correspond one-to-one to the resonances we observe in the BCS system.

In the damped pendulum, the Arnold tongue starts at a sharp value of the driving strength [40], satisfying the power
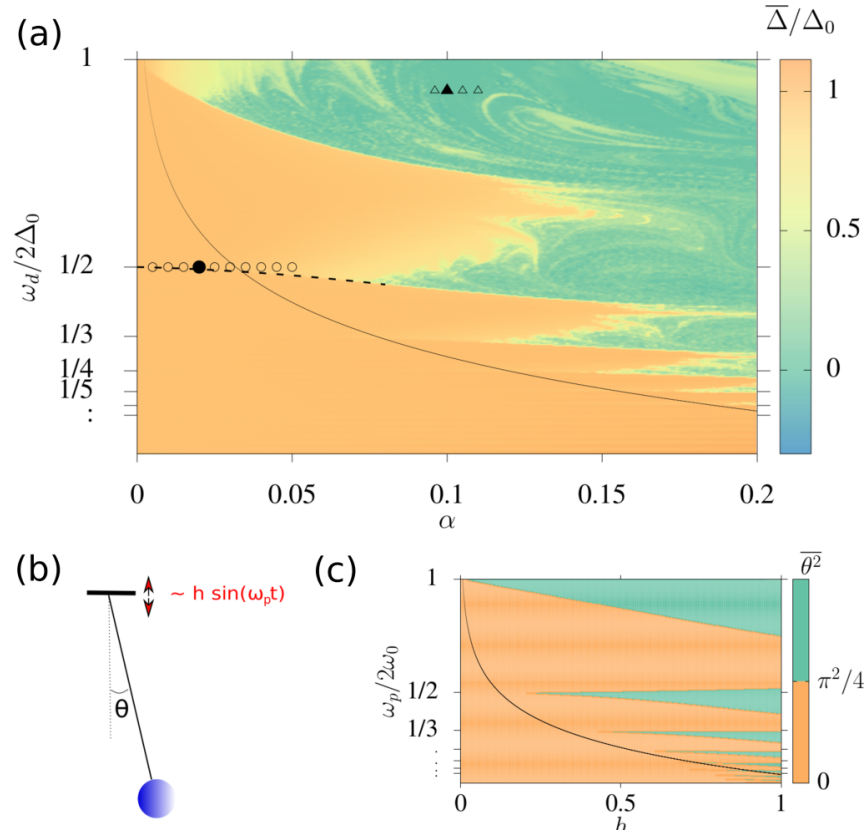

FIG. 1. (a) Dynamical phase diagram obtained as a false color plot of $\bar{\Delta}$ as a function of $\alpha$ and $\omega_{d}$. The order parameter was averaged in the interval $t \Delta_{0} \in[0,200]$. Parametric resonances occur at $2 \Delta_{0} / n$ with $n$ being a natural number similar to the vertically driven pendulum (b). The phase diagram of the latter is shown in panel (c) by plotting the mean-square amplitude of oscillation as a function of the pump frequency $\omega_{p}$ and the amplitude $h$ (in the harmonic approximation). The black curves in panels (a) and (c) show a power law delimiting the Arnold tongues in the presence of damping. Small discrepancies in panel (c) are finite time-window effects. In the case of the BCS system, we found that $\alpha=0.45 \eta^{\omega_{d}} / 2 \Delta_{0}$ with $\eta=0.005$ approximately describing the numerical results. The dashed black line in panel (a) indicates a weak first-order DPT. The open symbols indicate the parameters for which the rigidity of the Higgs mode (circles) and time crystal (triangles) has been checked [39].

law $h_{c}=\eta^{1 / n}$. This is approximately verified for the BCS system [thin black line in Figs. 1(a) and 1(c)] but the behavior is more complex. The upper boundary of the Arnold tongue has a fractal-like structure similar to the chaotic dynamics found in related systems [41,42]. In contrast, the lower boundary does not finish at the tip of the Arnold tongue but continues until $\alpha=0$ as a weak first-order DPT where $\bar{\Delta}$ changes discontinuously [Fig. 1(a), dashed line for $n=2$ ), producing a sharp edge.

Notice that, for simplicity, in the case of the pendulum we solved a linear model [39] and resonances appear as a divergence of the amplitude. In the case of a real pendulum, these divergences are cut off by nonlinearities. In the superconductor, the full nonlinearity of the BCS dynamics is included in the computations so analogous divergences are replaced by different nonlinear phenomena as discussed below.

To characterize the different dynamics outside and inside the Arnold tongues, in the following we analyze in more detail two representative examples. Figure 2 shows the dynamics away from the Arnold tongues [solid circle in Fig. 1(a)]. After some transient oscillations, the superconducting order parameter decreases in average and oscillates 

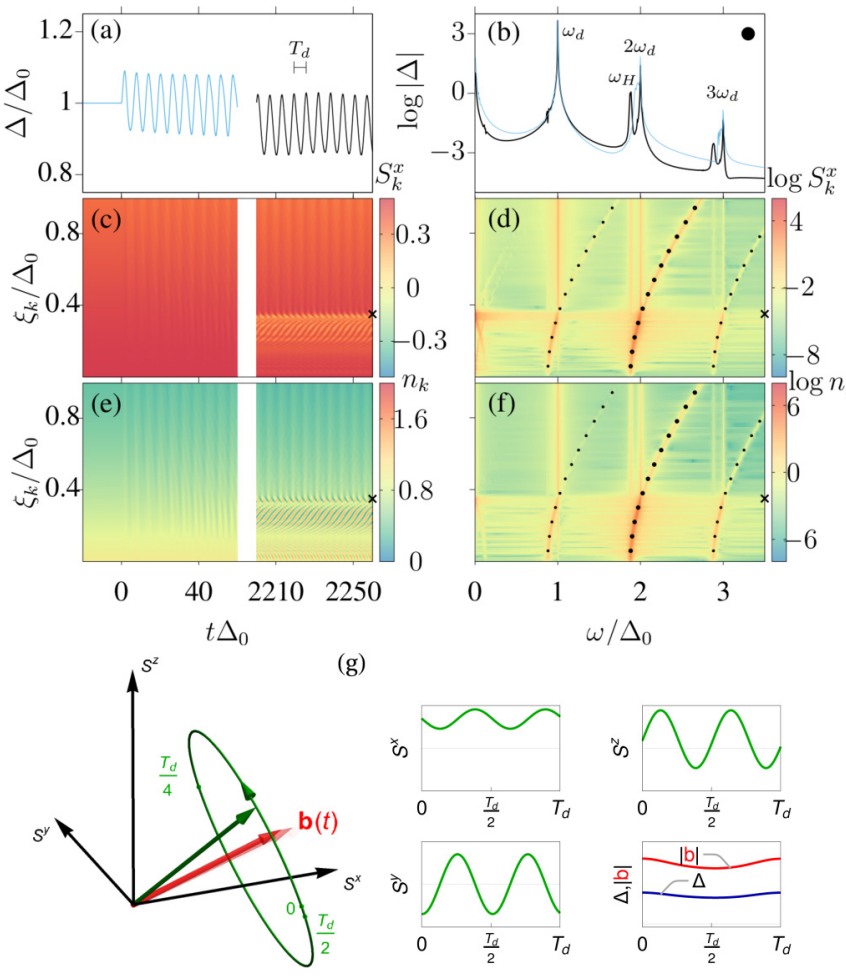

FIG. 2. The dynamics outside the Arnold tongues [corresponding to the solid circle in Fig. 1(a)] is characterized through $\Delta(t)$, $S_{k}^{x}(t)$, and $n_{k}(t)$. Panels (a), (c), and (e) show the transient dynamics (left) and the steady-state dynamics (right). Panels (b), (d), and (f) show the log of the FT. Black dots in panels (d) and (f) mark $\Omega_{L}$ and $\Omega_{L} \pm \omega_{d}$. The cross at the right of panels (c)-(f) indicate the boundary of the $2 \omega_{d}$-resonance regime. Panel $(\mathrm{g})$ illustrates schematically the pseudospin precessions around the pseudomagnetic field $\boldsymbol{b}(t)$, which has a time dependence through $\Delta(t)(\boldsymbol{k}$ label dropped for clarity). The chosen pseudospin with $\xi_{k} / \Delta_{0}=0.3$ is in the $2 \omega_{d}$-resonance regime, so its Larmor frequency is nearly twice the periodicity of $\Delta(t)$ and the modulus of $\boldsymbol{b}(t)$ as shown on the right.

around a new value, $\bar{\Delta}<\Delta_{0}$ [Fig. 2(a)]. We have also plotted the $x$ component of the pseudospin texture over time $S_{k}^{x}(t)$ [Fig. 2(c)], which is associated to the superconducting response through Eq. (2), as well as the time-dependent quasiparticle distribution $n_{k}(t)=1-2 S_{k}^{z}(t)$ [Fig. 2(e)]. The Fourier transforms (FT) in Figs. 2(d) and 2(f) show that the same set of frequencies appear in the dynamics of $n_{k}$ and $S_{k}^{x}$. As illustrated in Fig. 2(g), this is a simple consequence of the fact that pseudospins (green) make a tilted precession around the self-consistent pseudomagnetic field (red). As could be expected, the pseudospin Larmor frequency $\Omega_{L}$ is determined by the average gap (as opposed to the equilibrium gap). Indeed, the large dots in Figs. 2(d) and 2(f) mark $\omega=\Omega_{L}\left(\xi_{k}\right) \equiv 2 \sqrt{\xi_{k}^{2}+\bar{\Delta}^{2}}$. Also, Floquet sidebands appear at $\Omega_{L} \pm \omega_{d}$ (small dots).

The cross at the right of Figs. 2(c)-(f) indicate the value $\xi_{k}^{*}$ satisfying $2 \omega_{d}=\Omega_{L}\left(\xi_{k}^{*}\right)$. Quasiparticles with $\xi_{k} \lesssim \xi_{k}^{*}$ are driven strongly out of equilibrium, creating a sharp separation in $\xi_{k}$ among quasiparticles that respond strongly and weakly to the drive. We refer to this as $2 \omega_{d}$-resonant behavior to distinguish it from parametric resonances.
The peak at $\omega_{d}$ and higher harmonics, shown in Fig. 2(b), can be explained from linear response theory and weak nonlinearities. In contrast, an unexpected oscillation occurs with a frequency $\omega_{H}$ which is not commensurate with the driving frequency but instead satisfies $\omega_{H}=2 \bar{\Delta}$. Thus, it is an internal mode of the many-body system that spontaneously emerges in the dynamics (Higgs mode). Being the time analog of an incommensurate charge-density wave in a solid, these states are dubbed time quasicrystals in other contexts where they have been identified both experimentally [6] and theoretically $[22,43,44]$. Here, we find that its frequency is robust to changes in the drive [39] when measured in units of $2 \bar{\Delta}$ which is a general requirement defining time-crystal behavior. On the other hand, since a time crystal is often associated with a subharmonic response, we use the conventional denomination of "synchronized Higgs mode," keeping in mind that it shares many characteristics of time-crystal behavior.

The vertical features in Figs. 2(d) and 2(f) at $\omega=\omega_{H}$ reveal that the origin of the synchronized Higgs mode is not a simple consequence of the Van Hove singularity of the BCS density of states, but rather of a synchronization between a group of pseudospins. A similar oscillation emerges spontaneously in BCS quench protocols in which the attractive interaction is suddenly increased by a large amount [45-48]. There, also, the frequency of this Higgs mode is determined by the average gap [49]. Here, the synchronized Higgs mode emerges with a continuous wave pump and without the need of large driving amplitudes, a protocol which is much easier to implement experimentally [50].

Excitation of the Higgs mode by a periodic drive above the equilibrium gap was found in a layered Ginzburg-Landau model without quasiparticle excitations [22]. Our result applies to general BCS systems for driving frequency below the gap (where heating effects are expected to be minimized) and takes into account the full BCS dynamics including the effect of quasiparticle excitations.

We now switch to the typical dynamics inside the Arnold tongues (solid triangle in Fig. 1). Remarkably, we find that a new commensurate time-crystal condensate phase emerges. Indeed, as shown in Fig. 3, after a short transient, $\bar{\Delta}$ becomes zero [Fig. 3(a)] and the instantaneous order parameter oscillates with half of the drive frequency [Fig. 3(b)] as found in other models showing discrete time-translational symmetry breaking [4,5,29,51-57].

It has become customary to reserve the term "time crystal" for TTSB satisfying certain criteria $[28,58]$, which ensures a many-body origin. In our case, the terminology is justified by the fact that there is long-range order in time, in the sense that the symmetry breaking persists for at least thousands of Floquet cycles in our numerical calculations. In addition, we have checked [39] that the effect is not accidental, but there is "rigidity" in the TTSB. Changing the amplitude of the drive, the frequency response remains locked, which indicates a self-generated collective mode. Figure 3(d) shows that the subharmonic response is shared by a wide range of pseudospins as witnessed by the vertical feature at $\omega_{d} / 2$. The sum of all these contributions leads to the macroscopic TTSB response of the order parameter.

Figure 3(b) show that $\Delta(t)$ can be well approximated by only two Fourier components: $\Delta(t)=\Delta_{1} \cos \left(\omega_{d} t / 2\right)+$ 

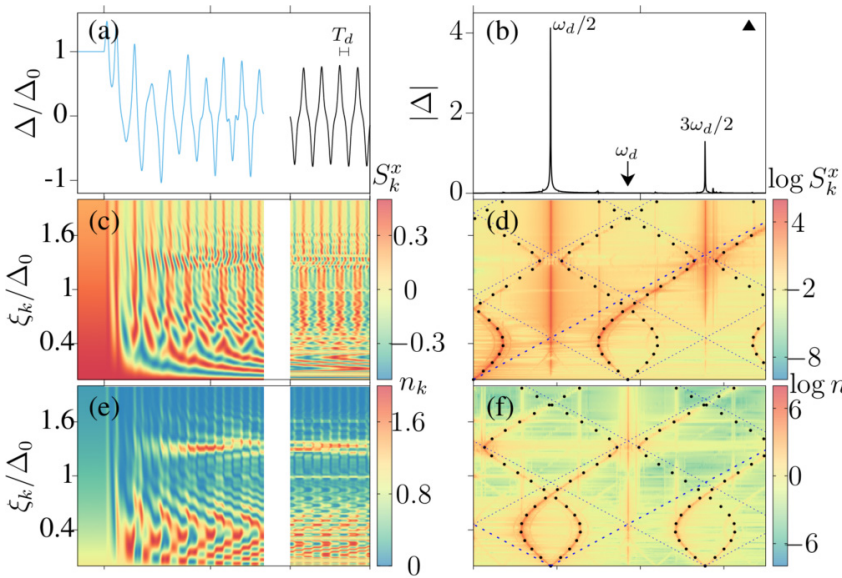

0

40
$t \Delta_{0}$
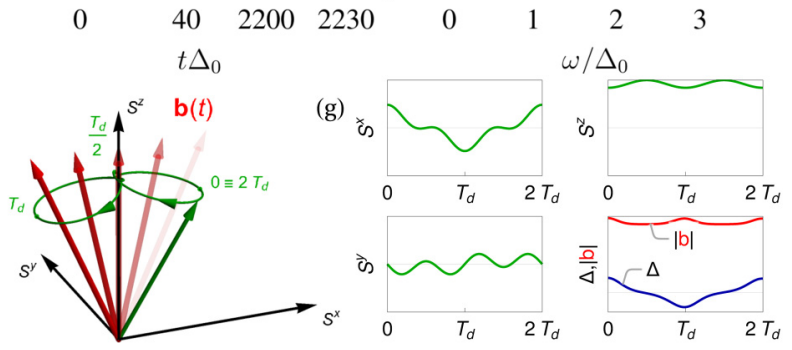

FIG. 3. Same as Fig. 2 but inside the Arnold tongue showing subharmonic dynamics [parameters corresponding to the solid triangle in Fig. 1(a)]. After a short transient, the average order parameter is driven to zero and oscillates with twice the periodicity of the drive [panels (a) and (g)]. Panel (g) schematizes the behavior of a quasiparticle with $\xi_{k} / \Delta_{0}=1.8$. The pseudomagnetic field (red) oscillates with a time-dependent length $|b|$ reminiscent of the vertically driven pendulum setting; i.e., the length is minimum each time the field is vertical $\left(t=T_{d} / 2,3 T_{d} / 2\right)$ (red line in the lower right panel). The pseudospin (green) precesses and follows $\boldsymbol{b}(t)$, making an " $\infty$-shaped" loop. $S^{x}$ has $2 T_{d}$ periodicity and self-consistently builds $\Delta(t)$ with the same periodicity. The dots in panel (d) are the result of a Floquet computation [39]. The dashed blue lines are the bare dispersion $\omega=\left|2 \xi_{k}\right|$ (more intense) and associated Floquet side bands $\left|2 \xi_{k} \pm n \omega_{d}\right|$, with $n=1,2,3, \ldots$ Notice that the Floquet spectrum appears shifted by $\omega_{d} / 2$ in the charge response (f) in relation to panel (d).

$\Delta_{2} \cos \left(3 \omega_{d} t / 2\right)$, with $\Delta_{1}=0.58 \Delta_{0}$ and $\Delta_{2}=0.156 \Delta_{0}$. Considering this self-consistent order parameter as an external drive acting on each pseudospin, it is possible to use the Floquet theory to compute analytically the spectrum of frequencies determining the dynamics [39]. This results in the dots shown in Figs. 3(d) and 3(f) which agree very well with the structures seen in the numerical simulations.

Near $\xi_{\mathbf{k}} / \Delta_{0} \approx 0.4$ a large gap of size $2 \Delta_{1}$ appears in the spectrum of Fig. 3(d) as the remnant of the superconducting pairing. However, rather than being centered at $\omega=0$ [as for a conventional superconducting phase, cf. Fig. 2(d)], it is centered at $\omega_{d} / 2$. Such a finite frequency gap can be understood as the avoiding crossing among the bare dispersion $\omega=\left|2 \xi_{\mathbf{k}}\right|$ and the first Floquet replica $\omega=\left|\omega_{d} \pm 2 \xi_{\mathbf{k}}\right|$ (dashed blue lines). Avoiding crossings involving higher Floquet replicas explains the smaller gaps of size $2 \Delta_{2}$ around $\xi_{k} / \Delta_{0} \approx 1.3$.

Figure $3(\mathrm{~g})$ schematizes the dynamics at high energy, where the subharmonic response is strong. Pseudospins pre- cess and follow the time-dependent field that they contribute to create. Because the $z$ axis coincides with a symmetry axis of the dynamics, different frequencies appear in the pairing [Fig. 3(d)] and charge [Fig. 3(f)] fluctuations, in contrast to Fig. 2 where such symmetry does not hold. Notice that charge fluctuations [Fig. 3(f)] respond at the drive frequency, in contrast to the subharmonic response of the pairing fluctuations [Fig. 3(d)].

Due to the gauge invariance of the equations, multiplying the real $\Delta(t)$ by an arbitrary time-independent phase factor, $\Delta(t) e^{i \phi}$, yields another solution of the time-dependent BCS problem. In other words, the dynamical phase breaks both discrete time-translational symmetry and U(1) symmetry. The latter symmetry breaking characterizes also an equilibrium BCS condensate; thus, the new state is dubbed a "commensurate time-crystal condensate."

For classical systems it has been proposed to use parametric oscillators as a building block of a time crystal [54,55]. In contrast, our building blocks, noninteracting pseudospins, do not show parametric resonances. The resonances and the timecrystal phase emerge as a result of the interactions between quasiparticles.

Heating and decoherence are often a concern for observing subtle out-of-equilibrium effects in condensates. As shown in Fig. S3 of Ref. [39], Arnold tongues are visible even if we restrict to relatively few decades of $t \Delta_{0}$. This can be compared with quasiparticle relaxation times $\tau$ of the order of microseconds measured in aluminium-based solid-state superconducting devices [59] $\left(\tau \Delta_{0} \approx 10^{6}\right)$. While the outof-equilibrium dynamics may strongly affect this coherence time, the gentle perturbation represented by a subgap drive suggests that the present effects could be observable in solidstate superconductors.

The phase diagram is also robust with respect to different driving methods. Indeed, we find similar results with a periodic drive of the density of states or driving with an external pairing field which will be shown elsewhere [60]. The former driving can be implemented with ultracold atoms, cavity QED, or $\mathrm{THz}$ radiation in condensed-matter systems with suitable polarizations [22,37,38,61-65].

Periodic $\lambda$-driving can be naturally implemented in ultracold atomic gases by using a small time-dependent magnetic field modulation in a Feshbach resonance. An alternative protocol has been implemented in cold fermionic lithium atoms [50]. Parametric resonances, time-crystal phases, and DPTs can be detected through magnetic sweep to the BEC side, giving access to the BCS condensed fraction and the instantaneous order parameter [50]. Additionally, in solid-state superconductors, a phonon-assisted $\lambda$-driving mechanism has been proposed [37] which could also be useful to test the prediction discussed here.

Very recently, an optical-cavity QED platform to simulate the BCS system has been proposed [66]. Such a setting is also promising to observe our predictions since it allows for a significant control of Hamiltonian parameters with long coherence times, as already demonstrated in related experiments $[67,68]$.

The BCS formalism, originally developed for superconductivity, also applies to weak-coupling charge- and 
spin-density waves. Therefore, our results are also relevant to these types of order at times short enough for the energy relaxation process to be neglected. Integrability breaking terms [47], residual many-body interactions, and coupling with a bath [69] should help us to understand the thermalization of the found dynamical phases and are an attractive topic for further studies.

Periodically driven dynamics have been studied in Josephson junction arrays with magnetic fields [70,71]. This is an interesting platform to search for parametric resonances and time-crystal features taking into account that such systems present also analogies with the driven pendulum.

To conclude, we have found that the dynamical phase diagram of a periodically driven BCS condensate is surprisingly rich: it shows Arnold tongues corresponding to parametric resonances mimicking the behavior of a vertically excited pendulum. The dynamics is highly nontrivial, showing commensurate (incommensurate) time-translational symmetry breaking inside (outside) the Arnold tongues. This calls for an experimental exploration of the phase diagram. Furthermore, our findings suggest exploring potential applications in parametric amplification, frequency converters, and sensing.

We are grateful for enlightening conversations with $\mathrm{C}$. Castellani and G. Seibold. We acknowledge financial support from the Italian MAECI and the Argentinian MINCYT through bilateral Project No. AR17MO7, from ANPCyT (Grants No. PICT 2016-0791 and No. PICT 2018-1509), from CONICET (Grant No. PIP 11220150100506CO), from SeCyT-UNCuyo (Grant No. 06/C603), from the Italian Ministry for University and Research through PRIN Projects No. 2017Z8TS5B and No. 20207ZXT4Z, and from Regione Lazio (L.R. 13/08) under project SIMAP. H.P.O.C. is supported by a Marie Skłodowska-Curie Individual Fellowship, Grant No. SUPERDYN 893743
[1] A. Zenesini, H. Lignier, D. Ciampini, O. Morsch, and E. Arimondo, Coherent Control of Dressed Matter Waves, Phys. Rev. Lett. 102, 100403 (2009).

[2] J. Struck, C. Ölschläger, R. Le Targat, P. Soltan-Panahi, A. Eckardt, M. Lewenstein, P. Windpassinger, and K. Sengstock, Quantum simulation of frustrated classical magnetism in triangular optical lattices, Science 333, 996 (2011).

[3] J. Struck, M. Weinberg, C. Ölschläger, P. Windpassinger, J. Simonet, K. Sengstock, R. Höppner, P. Hauke, a. Eckardt, M. Lewenstein, and L. Mathey, Engineering Ising-XY spin-models in a triangular lattice using tunable artificial gauge fields, Nat. Phys. 9, 738 (2013).

[4] J. Zhang, P. W. Hess, A. Kyprianidis, P. Becker, A. Lee, J. Smith, G. Pagano, I.-D. Potirniche, A. C. Potter, A. Vishwanath, N. Y. Yao, and C. Monroe, Observation of a discrete time crystal, Nature (London) 543, 217 (2017).

[5] S. Choi, J. Choi, R. Landig, G. Kucsko, H. Zhou, J. Isoya, F. Jelezko, S. Onoda, H. Sumiya, V. Khemani, C. Von Keyserlingk, N. Y. Yao, E. Demler, and M. D. Lukin, Observation of discrete time-crystalline order in a disordered dipolar many-body system, Nature (London) 543, 221 (2017).

[6] S. Autti, V. B. Eltsov, and G. E. Volovik, Observation of a Time Quasicrystal and Its Transition to a Superfluid Time Crystal, Phys. Rev. Lett. 120, 215301 (2018).

[7] A. Kyprianidis, F. Machado, W. Morong, P. Becker, K. S. Collins, D. V. Else, L. Feng, P. W. Hess, C. Nayak, G. Pagano, N. Y. Yao, and C. Monroe, Observation of a prethermal discrete time crystal, Science 372, 1192 (2021).

[8] D. Fausti, R. I. Tobey, N. Dean, S. Kaiser, A. Dienst, M. C. Hoffmann, S. Pyon, T. Takayama, H. Takagi, and A. Cavalleri, Light-induced superconductivity in a stripe-ordered cuprate, Science 331, 189 (2011).

[9] M. Mitrano, A. Cantaluppi, D. Nicoletti, S. Kaiser, A. Perucchi, S. Lupi, P. Di Pietro, D. Pontiroli, M. Riccò, S. R. Clark, D. Jaksch, and A. Cavalleri, Possible light-induced superconductivity in $\mathrm{K}_{3} \mathrm{C}_{60}$ at high temperature, Nature (London) 530, 461 (2016).

[10] M. Beck, I. Rousseau, M. Klammer, P. Leiderer, M. Mittendorff, S. Winnerl, M. Helm, G. N. Gol'tsman, and J. Demsar, Transient Increase of the Energy Gap of Supercon- ducting NbN Thin Films Excited by Resonant Narrow-Band Terahertz Pulses, Phys. Rev. Lett. 110, 267003 (2013).

[11] T. F. Nova, A. S. Disa, M. Fechner, and A. Cavalleri, Metastable ferroelectricity in optically strained $\mathrm{SrTiO}_{3}$, Science 364, 1075 (2019).

[12] A. Kogar, A. Zong, P. E. Dolgirev, X. Shen, J. Straquadine, YaQing Bie, X. Wang, T. Rohwer, I.-C. Tung, Y. Yang, R. Li, J. Yang, S. Weathersby, S. Park, M. E. Kozina, E. J. Sie, H. Wen, P. Jarillo-Herrero, I. R. Fisher, X. Wang et al., Light-induced charge density wave in $\mathrm{LaTe}_{3}$, Nat. Phys. 16, 159 (2020).

[13] D. N. Basov, R. D. Averitt, and D. Hsieh, Towards properties on demand in quantum materials, Nat. Mater. 16, 1077 (2017).

[14] G. Floquet, Sur les équations différentielles linéaires à coefficients périodiques, Annales scientifiques de l'É.N.S. 2e série 12, 47 (1883).

[15] T. Oka and S. Kitamura, Floquet engineering of quantum materials, Annu. Rev. Condens. Matter Phys. 10, 387 (2019).

[16] R. Ma, B. Saxberg, C. Owens, N. Leung, Y. Lu, J. Simon, and D. I. Schuster, A dissipatively stabilized Mott insulator of photons, Nature (London) 566, 51 (2019).

[17] M. A. Castellanos-Beltran, K. D. Irwin, G. C. Hilton, L. R. Vale, and K. W. Lehnert, Amplification and squeezing of quantum noise with a tunable Josephson metamaterial, Nat. Phys. 4, 929 (2008).

[18] N. Bergeal, F. Schackert, M. Metcalfe, R. Vijay, V. E. Manucharyan, L. Frunzio, D. E. Prober, R. J. Schoelkopf, S. M. Girvin, and M. H. Devoret, Phase-preserving amplification near the quantum limit with a Josephson ring modulator, Nature (London) 465, 64 (2010).

[19] C. Macklin, K. O'Brien, D. Hover, M. E. Schwartz, V. Bolkhovsky, X. Zhang, W. D. Oliver, and I. Siddiqi, A nearquantum-limited Josephson traveling-wave parametric amplifier, Science 350, 307 (2015).

[20] B. H. Eom, P. K. Day, H. G. Leduc, and J. Zmuidzinas, A wideband, low-noise superconducting amplifier with high dynamic range, Nat. Phys. 8, 623 (2012).

[21] S. Rajasekaran, E. Casandruc, Y. Laplace, D. Nicoletti, G. D. Gu, S. R. Clark, D. Jaksch, and A. Cavalleri, Parametric amplification of a superconducting plasma wave, Nat. Phys. 12, 1012 (2016). 
[22] G. Homann, J. G. Cosme, and L. Mathey, Higgs time crystal in a high- $T_{c}$ superconductor, Phys. Rev. Research 2, 043214 (2020).

[23] A. Von Hoegen, M. Fechner, M. Först, N. Taherian, E. Rowe, A. Ribak, J. Porras, B. Keimer, M. Michael, E. Demler, and A. Cavalleri, Parametrically amplified phase-incoherent superconductivity in $\mathrm{YBa}_{2} \mathrm{Cu}_{3} \mathrm{O}_{6+x}$, arXiv:1911.08284 .

[24] M. Buzzi, G. Jotzu, A. Cavalleri, J. I. Cirac, E. A. Demler, B. I. Halperin, M. D. Lukin, T. Shi, Y. Wang, and D. Podolsky, Higgs-Mediated Optical Amplification in a Nonequilibrium Superconductor, Phys. Rev. X 11, 011055 (2021).

[25] H. Y. Liu, I. Gierz, J. C. Petersen, S. Kaiser, A. Simoncig, A. L. Cavalieri, C. Cacho, I. C. E. Turcu, E. Springate, F. Frassetto, L. Poletto, S. S. Dhesi, Z.-A. Xu, T. Cuk, R. Merlin, and A. Cavalleri, Possible observation of parametrically amplified coherent phasons in $\mathrm{K}_{0.3} \mathrm{MoO}_{3}$ using time-resolved extremeultraviolet angle-resolved photoemission spectroscopy, Phys. Rev. B 88, 045104 (2013).

[26] A. Cartella, T. F. Nova, M. Fechner, R. Merlin, and A. Cavalleri, Parametric amplification of optical phonons, Proc. Natl. Acad. Sci. USA 115, 12148 (2018).

[27] F. Wilczek, Quantum Time Crystals, Phys. Rev. Lett. 109, 160401 (2012).

[28] N. Y. Yao, A. C. Potter, I.-D. Potirniche, and A. Vishwanath, Discrete Time Crystals: Rigidity, Criticality, and Realizations, Phys. Rev. Lett. 118, 030401 (2017).

[29] A. Russomanno, F. Iemini, M. Dalmonte, and R. Fazio, Floquet time crystal in the Lipkin-Meshkov-Glick model, Phys. Rev. B 95, 214307 (2017).

[30] B. Huang, Y.-H. Wu, and W. V. Liu, Clean Floquet Time Crystals: Models and Realizations in Cold Atoms, Phys. Rev. Lett. 120, 110603 (2018).

[31] R. Khasseh, R. Fazio, S. Ruffo, and A. Russomanno, ManyBody Synchronization in a Classical Hamiltonian System, Phys. Rev. Lett. 123, 184301 (2019).

[32] A. Pizzi, D. Malz, G. De Tomasi, J. Knolle, and A. Nunnenkamp, Time crystallinity and finite-size effects in clean Floquet systems, Phys. Rev. B 102, 214207 (2020).

[33] A. Pizzi, J. Knolle, and A. Nunnenkamp, Higher-order and fractional discrete time crystals in clean long-range interacting systems, Nat. Commun. 12, 2341 (2021).

[34] V. K. Kozin and O. Kyriienko, Quantum Time Crystals from Hamiltonians with Long-Range Interactions, Phys. Rev. Lett. 123, 210602 (2019).

[35] X. Yang and Zi Cai, Dynamical Transitions and Critical Behavior between Discrete Time Crystal Phases, Phys. Rev. Lett. 126, 020602 (2021).

[36] P. W. Anderson, Random-phase approximation in the theory of superconductivity, Phys. Rev. 112, 1900 (1958).

[37] H. P. Ojeda Collado, J. Lorenzana, G. Usaj, and C. A. Balseiro, Population inversion and dynamical phase transitions in a driven superconductor, Phys. Rev. B 98, 214519 (2018).

[38] H. P. Ojeda Collado, G. Usaj, J. Lorenzana, and C. A. Balseiro, Nonlinear dynamics of driven superconductors with dissipation, Phys. Rev. B 101, 054502 (2020).

[39] See Supplemental Material at http://link.aps.org/supplemental/ 10.1103/PhysRevResearch.3.L042023 for numerical simulation details, the demonstration of the rigidity of time-crystal and Higgs mode, the Floquet theory computation and a discussion on the phase diagram at short times.
[40] L. D. Landau and E. M. Lifshitz, Mechanics, Course of Theoretical Physics Vol. 1 (Butterworth-Heinenann, Oxford, 1976).

[41] A. Lerose, J. Marino, B. Žunkovič, A. Gambassi, and A. Silva, Chaotic Dynamical Ferromagnetic Phase Induced by Nonequilibrium Quantum Fluctuations, Phys. Rev. Lett. 120, 130603 (2018).

[42] A. Lerose, B. Žunkovič, J. Marino, A. Gambassi, and A. Silva, Impact of nonequilibrium fluctuations on prethermal dynamical phase transitions in long-range interacting spin chains, Phys. Rev. B 99, 045128 (2019).

[43] K. Giergiel, A. Kuroś, and K. Sacha, Discrete time quasicrystals, Phys. Rev. B 99, 220303(R) (2019).

[44] G. E. Volovik, On the broken time translation symmetry in macroscopic systems: Precessing states and off-diagonal longrange order, JETP Lett. 98, 491 (2013).

[45] R. A. Barankov, L. S. Levitov, and B. Z. Spivak, Collective Rabi Oscillations and Solitons in a Time-Dependent BCS Pairing Problem, Phys. Rev. Lett. 93, 160401 (2004).

[46] R. A. Barankov and L. S. Levitov, Synchronization in the BCS Pairing Dynamics as a Critical Phenomenon, Phys. Rev. Lett. 96, 230403 (2006).

[47] J. A. Scaramazza, P. Smacchia, and E. A. Yuzbashyan, Consequences of integrability breaking in quench dynamics of pairing Hamiltonians, Phys. Rev. B 99, 054520 (2019).

[48] A. Gambassi and P. Calabrese, Quantum quenches as classical critical films, Europhysics Lett. 95, 66007 (2011).

[49] G. Seibold and J. Lorenzana, Nonequilibrium dynamics from BCS to the bosonic limit, Phys. Rev. B 102, 144502 (2020).

[50] A. Behrle, T. Harrison, J. Kombe, K. Gao, M. Link, J.S. Bernier, C. Kollath, and M. Köhl, Higgs mode in a strongly interacting fermionic superfluid, Nat. Phys. 14, 781 (2018).

[51] K. Sacha, Modeling spontaneous breaking of time-translation symmetry, Phys. Rev. A 91, 033617 (2015).

[52] D. V. Else, B. Bauer, and C. Nayak, Floquet Time Crystals, Phys. Rev. Lett. 117, 090402 (2016).

[53] A. Chandran and S. L. Sondhi, Interaction-stabilized steady states in the driven $O(N)$ model, Phys. Rev. B 93, 174305 (2016).

[54] T. L. Heugel, M. Oscity, A. Eichler, O. Zilberberg, and R. Chitra, Classical Many-Body Time Crystals, Phys. Rev. Lett. 123, 124301 (2019).

[55] N. Y. Yao, C. Nayak, L. Balents, and M. P. Zaletel, Classical discrete time crystals, Nat. Phys. 16, 438 (2020).

[56] M. Natsheh, A. Gambassi, and A. Mitra, Critical properties of the Floquet time crystal within the Gaussian approximation, Phys. Rev. B 103, 014305 (2021).

[57] M. Natsheh, A. Gambassi, and A. Mitra, Critical properties of the prethermal Floquet time crystal, Phys. Rev. B 103, 224311 (2021).

[58] D. V. Else, C. Monroe, C. Nayak, and N. Y. Yao, Discrete time crystals, Annu. Rev. Condens. Matter Phys. 11, 467 (2020).

[59] O. P. Saira, A. Kemppinen, V. F. Maisi, and J. P. Pekola, Vanishing quasiparticle density in a hybrid $\mathrm{Al} / \mathrm{Cu} / \mathrm{Al}$ single-electron transistor, Phys. Rev. B 85, 012504 (2012).

[60] H. P. Ojeda Collado, G. Usaj, C. A. Balseiro, D. H. Zanette, and J. Lorenzana, Dynamical phase transitions in driven BCS systems (unpublished). 
[61] R. Matsunaga, Y. I. Hamada, K. Makise, Y. Uzawa, H. Terai, Z. Wang, and R. Shimano, Higgs Amplitude Mode in the BCS Superconductors $\mathrm{Nb}_{1-x} \mathrm{Ti}_{x} \mathrm{~N}$ Induced by Terahertz Pulse Excitation, Phys. Rev. Lett. 111, 057002 (2013).

[62] R. Matsunaga, N. Tsuji, H. Fujita, A. Sugioka, K. Makise, Y. Uzawa, H. Terai, Z. Wang, H. Aoki, and R. Shimano, Lightinduced collective pseudospin precession resonating with Higgs mode in a superconductor, Science 345, 1145 (2014).

[63] T. Cea, C. Castellani, G. Seibold, and L. Benfatto, Nonrelativistic Dynamics of the Amplitude (Higgs) Mode in Superconductors, Phys. Rev. Lett. 115, 157002 (2015).

[64] R. Shimano and N. Tsuji, Higgs mode in superconductors, Annu. Rev. Condens. Matter Phys. 11, 103 (2020).

[65] N. Tsuji and H. Aoki, Theory of Anderson pseudospin resonance with Higgs mode in superconductors, Phys. Rev. B 92, 064508 (2015).

[66] R. J. Lewis-Swan, D. Barberena, J. R. K. Cline, D. J. Young, J. K. Thompson, and A. M. Rey, Cavity-QED Quantum Simulator of Dynamical Phases of a Bardeen-Cooper-Schrieffer Superconductor, Phys. Rev. Lett. 126, 173601 (2021).
[67] M. A. Norcia, R. J. Lewis-Swan, J. R. K. Cline, B. Zhu, A. M. Rey, and J. K Thompson, Cavity-mediated collective spin-exchange interactions in a strontium superradiant laser, Science 361, 259 (2018).

[68] J. A. Muniz, D. Barberena, R. J. Lewis-Swan, D. J. Young, J. R. K. Cline, A. M. Rey, and J. K. Thompson, Exploring dynamical phase transitions with cold atoms in an optical cavity, Nature (London) 580, 602 (2020).

[69] H. P. Ojeda Collado, G. Usaj, J. Lorenzana, and C. A. Balseiro, Fate of dynamical phases of a BCS superconductor beyond the dissipationless regime, Phys. Rev. B 99, 174509 (2019).

[70] C. Reichhardt, A. B. Kolton, D. Domínguez, and N. GrønbechJensen, Phase-locking of driven vortex lattices with transverse ac force and periodic pinning, Phys. Rev. B 64, 134508 (2001).

[71] V. I. Marconi, A. B. Kolton, D. Domínguez, and N. GrønbechJensen, Transverse phase locking in fully frustrated Josephson junction arrays: A different type of fractional giant steps, Phys. Rev. B 68, 104521 (2003). 